\section{Area: Medicina Baseada em Euidencias \\ QUAL A EFICÁCIA DAS MICROMOLAS INTRACEREBRAIS EM RELAÇÃO AO PROCEDIMENTO DE CLIPAGEM CIRÚRGICA NOS ANEURISMAS CEREBRAIS ROTOS?}

Muitos aneurismas intracranianos são assintomáticos, sendo que, provavelmente, só serão detectados como achado de exames de imagem. Outros podem gerar sintomas por compressão de estruturas cerebrais vizinhas, ou por causarem hemorragia subaracnoidéia após romperem. A mortalidade do aneurisma roto (hemorragia maciça, re-sangramento e isquemia cerebral) está entre $40 \%$ a $50 \%$, com quadros de morbidade severa de $10 \%$ a $20 \%$. O risco de rotura estimado é de 1,9\% por ano, sendo fatores de risco: sexo feminino, tabagismo, uso de cocaína, sintomáticos, hipertensão arterial, maior que $10 \mathrm{~mm}$, ou localizados na circulação posterior. As formas principais de tratamento são: a observação, a cirurgia e a embolização com micromolas. A observação tem sido considerada em aneurismas pequenos $(<10 \mathrm{~mm})$, em pacientes assintomáticos. A cirurgia (clipagem ou ligadura) pode ser precoce (nos primeiros dias), ou tardia. A cirurgia precoce tem como vantagem, a introdução de medicação anti-hipertensiva apropriada, e como desvantagens, o risco excessivo operatório (tecido cerebral friável, distúrbio cerebral autoregulatório), e na segunda semana, risco elevado de isquemia. A cirurgia tardia tem como vantagem, as melhores condições operatórias e o paciente mais estável, e como desvantagem, o risco de re-sangramento e a restrição à medicação anti-hipertensiva (vasoespasmo). Não há diferença nos resultados (morte ou dependência) quando a cirurgia é realizada precocemente (0-3 dias) ou tardiamente (+8dias). A embolização (micromolas) está contra-indicada, principalmente, em aneurismas maiores do que $10 \mathrm{~mm}$ ou com pescoço (colo) maior ou igual a $4 \mathrm{~mm}$, aneurismas com índice fundo (cúpula) / pescoço (colo) menor do que 1 e aneurismas na bifurcação da artéria cerebral média. 0 índice de complicações varia de 8,6\% a 18,6\%, sendo evento tromboembólico (2,5\% a 14,5\%), perfuração do aneurisma $(2,3 \%$ a $4,7 \%)$, ou migração das molas $(0,5 \%$ a $3 \%)$. Para a avaliação dos pacientes pós-tratamento são utilizadas escalas: Hunt-Hess Scale; World Federation of Neurosurgeons (WFNS) SAH Scale; Modified Glasgow coma Scale.

\section{Estratégia de busca e seleção da evidência:}

$\mathbf{P}$ - paciente adulto, com hemorragia subaracnóidea e aneurisma roto (artéria comunicante anterior), diagnosticado por arteriografia.

I - Introdução de micromolas (hemodinâmica)

C - clipagem cirúrgica

O - Morte ou recorrência.

As bases primárias consultadas foram: Medline, Embase, Lilacs, Cochrane e as Bases de Avaliação Tecnológica consultadas foram: NHS Health Technology Assessment Programme; Canadian Agency for Drugs and Technologies in Health e AHCPR Archived Technology Assessments. A estratégia de busca utilizada nas bases primárias foi: ("Embolization, Therapeutic"[MeSH] NOT "Chemoembolization, Therapeutic"[MeSH]) AND (coil*) AND ("Intracranial Aneurysm"[MeSH]). Foram utilizados os limites Randomized Controlled Trial e Systematic Reviews. Após avaliação crítica das evidências obtidas, foram selecionados uma revisão sistemática ${ }^{1}$, sem meta-análise e três $\mathrm{ECR}^{2-5,8}$. Nas bases de avaliação tecnológica foram recuperadas e selecionadas duas avaliações ${ }^{6,7}$.

\section{Síntese da evidência disponível}

Em pacientes, com quadro de hemorragia subaracnóidea; principalmente, clinicamente classificados, pela World Federation of Neurosurgeons (WFNS) Scale, em grau $\leq 2$; com ruptura de aneurisma cerebral, principalmente único, na circulação anterior e menor do que 10 mm de diâmetro, a embolização com micromolas; nos primeiros 28 dias do evento, quando comparada com a neurocirurgia (clipagem do aneurisma), produz os seguintes efeitos:

Reduz a mortalidade e a dependência (pela Escala Rankin modificada de 3-5), no primeiro ano, em 7,4\% dos pacientes tratados - NNT: 13 , e no quinto ano apenas a mortalidade em 3,1\% (NNT32)

Apresenta o mesmo número de re-sangramentos no primeiro ano, entretanto, apesar de pequeno número de re-sangramentos em cinco anos, aumenta o risco em 0,3\% (NNH143);

O custo da embolização é menor em relação à duração do procedimento, custo profissional, tempo de hospitalização, tempo de rehabilitação e retorno mais rápido às atividades.

O custo da embolização é maior em relação ao custo total e do material.

Wanderley Marques Bernardo FRANCISCO LIMA ${ }^{2}$

Luca Silveira Bernardo 3

1. Coordenador do Projeto Diretrizes AMB-CFM e Professor de Medicina Baseada em Evidência da Faculdade de Medicina de Santos - UNILUS, Santos,SP

2. Câmara Técnica de Medicina Baseada em Evidência da Federação Unimed do Estado Rio de Janeiro, RJ

3. Acadêmico da Faculdade de Medicina de Santos - UNILUS, Santos, SP

\section{Referências}

1.Van der Schaaf I, Algra A, Wermer M, Molyneux A, Clarke M, van Gijn J, et al. Endovascular coiling versus neurosurgical clipping for patients with aneurysmal subarachnoid haemorrhage. Cochrane Database Syst Rev 2005 Oct 19;(4):CD003085.

2. Molyneux AJ, Kerr RS, Yu LM, Clarke M, Sneade M, Yarnold JA, Sandercock P; International Subarachnoid Aneurysm Trial (ISAT) Collaborative Group. International subarachnoid aneurysm trial (ISAT) of neurosurgical clipping versus endovascular coiling in 2143 patients with ruptured intracranial aneurysms: a randomised comparison of effects on survival, dependency, seizures, rebleeding, subgroups, and aneurysm occlusion. Lancet 2005; 366:809-17. 


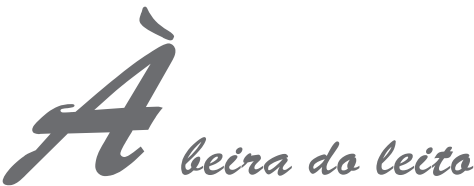

3. Molyneux A, Kerr R, Stratton I, Sandercock P, Clarke M, Shrimpton J, Holman R; International Subarachnoid Aneurysm Trial (ISAT) Collaborative Group. International Subarachnoid Aneurysm Trial (ISAT) of neurosurgical clipping versus endovascular coiling in 2143 patients with ruptured intracranial aneurysms: a randomised trial. Lancet 2002; 360:1267-74.

4.Bairstow P, Dodgson A, Linto J, Khangure M. Comparison of cost and outcome of endovascular and neurosurgical procedures in the treatment of ruptured intracranial aneurysms. Australas Radiol 2002; 46:249-51.

5.Vanninen R, Koivisto T, Saari T, Hernesniemi J, Vapalahti M. Ruptured intracranial aneurysms: acute endovascular treatment with electrolytically detachable coils--a prospective randomized study. Radiology 1999; 211:325-36.
6. Ferrante D. Coil embolization vs conventional surgery for the management of brain aneurysms. Ciudad de Buenos Aires: Institute for Clinical Effectiveness and Health Policy (IECS) 2004. Institute for Clinical Effectiveness and Health Policy (IECS).

7.Coil Embolization for Intracranial Aneurysms. The Health Technology Assessment Program. Canadian Agency for Drugs and Technologies in Health. Completed March 2004. Updated January 2006. Ontario. Canada.

8. Molyneux AJ, Kerr RS, Birks J, Ramzi N, Yarnold J, Sneade M, Rischmiller J; ISAT Collaborators. Risk of recurrent subarachnoid haemorrhage, death, or dependence and standardised mortality ratios after clipping or coiling of an intracranial aneurysm in the International Subarachnoid Aneurysm Trial (ISAT): long-term follow-up. Lancet Neurol 2009; 8: 427-33. 